\title{
Prevalence and Antibiogram of Generic Extended-Spectrum $\beta$-Lactam-Resistant Enterobacteria in Healthy Pigs
}

\author{
Ifeoma C. UGWU ${ }^{1}$, Madubuike U. ANYANWU ${ }^{2 *}$, Chidozie C. UGWU ${ }^{3}$, \\ Ogbonna W. UGWUANYI ${ }^{1}$ \\ ${ }^{1}$ University of Nigeria, Faculty of Veterinary Medicine, Department of Veterinary Pathology and Microbiology, Nsukka; \\ ifeoma.cugwn@unn.edu.ng;fredbanyi@gmail.com \\ ${ }^{2}$ University of Nigeria, Microbiology Unit, Department of Veterinary Pathology and Microbiology, Nsukka, Nigeria; \\ madubuike.anyanun@unn.edu.ng (*orrespondingauthor) \\ ${ }^{3}$ Federal University of Technology, Department of Animal Science and Technology, Owerri, Nigeria; chidozie.ugwu@futo.edu.ng
}

\begin{abstract}
This study was conducted to isolate generic extended-spectrum $\beta$-lactam (ESBL)-resistant enterobacteria from pigs reared in Enugu State Southeast, Nigeria and determine the antibacterial resistance profile of the isolates. Rectal swabs were collected from 190, randomly selected, apparently healthy pigs. Isolation of ESBL-resistant enterobacteria was done using Mac Conkey agar supplemented with $2 \mu \mathrm{g} / \mathrm{ml}$ of cefotaxime. Phenotypic characterization of the isolates to generic level was done following standard biochemical methods. Phenotypic resistance of the isolates to antibacterial agents was determined using the disc diffusion method. Out of 46 ESBL-resistant enterobacterial isolates, 4 (8.7\%) were Escherichia coli, 11 (23.9\%) were Salmonella species, while 31 (67.4\%) were Klebsiella species. Resistance of the Salmonella isolates was $45.5 \%$ to ciprofloxacin, $36.4 \%$ to ofloxacin and levofloxacin, $9.1 \%$ to norfloxacin, amikacin and gentamicin, $27.3 \%$ to streptomycin, $72.7 \%$ to chloramphenicol and $90.9 \%$ to tetracycline. Resistance of the Klebsiella isolates was $93.5 \%$ to ampicillin, $12.9 \%$ to ciprofloxacin, $19.4 \%$ to ofloxacin and levofloxacin, $9.7 \%$ to norfloxacin and streptomycin, $64.5 \%$ to chloramphenicol and $38.7 \%$ to tetracycline. Resistance of the E. coli isolates was $100 \%$ to gentamicin, $75 \%$ to ampicillin and streptomycin, $50 \%$ to ciprofloxacin, norfloxacin, chloramphenicol and tetracycline, and $25 \%$ to ofloxacin, levofloxacin and amikacin. All the isolates were resistant to ceftriaxone, cefotaxime, ceftazidime, cefepime, cefpodoxime, amoxicillin/clavulanic acid and aztreonam. Resistance of the isolates to more than 3 classes of antibacterial agents tested was $54.8 \%$ for Klebsiella, $90.9 \%$ for Salmonella and 100\% for E. coli, respectively. This study has shown that pigs reared in Enugu State Southeast, Nigeria, are colonized by ESBL-resistant Enterobactericeae and are potential reservoirs and disseminators of these organisms.
\end{abstract}

Keywords: antibiogram, $\beta$-lactam-resistant, Enterobacteriaceae, extended-spectrum, porcine

\section{Introduction}

Antimicrobial resistance has recently been identified as one of the greatest threat to human health and developing countries, including Nigeria, are worst hit by this crisis (WHO, 2014). Extensive use of antibacterial agents in foodproducing animals has been rated a major cause of this crisis (Geser et al., 2012). The situation is worsened by indiscriminate use of antibacterial agents, especially $\beta$-lactams, in sub-therapeutic/therapeutic doses for growth promotion, prophylaxis and treatment of bacterial diseases in foodproducing animals (Waters et al., 2011). Developing countries, including Nigeria, are major culprits due to the lack of strict policy regarding the use of antibacterial agents in humans and food-producing animals (Wall, 2014). This has led to increased resistance to most of the antibacterial agents (especially the $\beta$-lactams) (CLSI, 2012). Enterobacteriaceae constitute normal bacterial flora in intestinal tract of foodproducing animals and humans (Ben Sallem et al., 2012). Indiscriminate use of $\beta$-lactams (particularly 3rd- [e.g. cefotaxime, ceftriaxone, cefpodoxime, ceftazidime] and 4th[e.g. cefepime] generation cephalosporins) has led to selection pressure and development of resistance to these drugs by enterobacteria (Geser et al., 2011). These $3^{\text {rd }}$ and $4^{\text {th }}$ generation cephalosporins are extended-spectrum $\beta$-lactams (ESBL) whose resistance in enterobacteria is mediated by extended-spectrum beta-lactamases (ESBLs) (Garcia-Graells et al., 2010; Geser et al., 2011).

Extended spectrum $\beta$-lactam (ESBL)-resistant enterobacteria also exhibit resistance to many other classes of antibacterial agents including aminoglycosides, fluoroquinolones, phenicols and potentiated sulfonamides (Gniadikowski, 2001; Carattoli, 2008; Geser et al., 2012). Thus, ESBL-resistant enterobacterial isolates are multidrug 
resistant (Li et al., 2014). This multidrug resistance accounts for the limited therapeutic options available for treating infections associated with ESBL-resistant enterobacteria (Blaak et al., 2014). ESBL-resistant enterobacteria are the most prevalent causes of nosocomial and communityacquired infections globally (Li et al., 2014). These infections are often fatal due to limited therapeutic options, while the treatment option is using the last resort drug carbapenems (Canton et al., 2012; Blaak et al., 2014). This has also resulted in the recent increasing numbers of enterobacterial resistance to the carbapenems (Zurflu et al., 2013; Blaak et al., 2014). Therefore, determination of antibacterial resistance profile of ESBL-resistant enterobacteria is useful for empirical treatment of infections associated with such organisms.

As commensal organisms in the intestinal tract, ESBLresistant enterobacteria harboured by food-producing animals could be discharged into the environment, thereby serving as disseminators of genes encoding for $\beta$-lactam resistance. Beta-lactam resistance genes present in the discharged enterobacteria could be acquired by horizontal transfer to pathogenic human bacteria, thereby complicating infections and compromising antibacterial therapy (GarciaGraells et al., 2010). Presence of ESBL-resistant enterobacteria in faecal samples of food-producing animals represents a risk for carcass contamination at slaughter and subsequent potential for contamination of retail meat products (Geser et al., 2011). This contamination risk is particularly important in developing countries, such as Nigeria, where inadequate hygienic practices are employed during meat processing. Consumption of contaminated meat results in colonization of humans with ESBL-resistant bacteria, jeopardizing subsequent antibacterial therapies in carriers (Geser et al., 2011).

Isolation of ESBL-resistant enterobacteria in meat and meat products raised questions about the presence of ESBLresistant organisms in food-producing animals (Jensen et al., 2006; Jouini et al., 2007; Hammad et al., 2008; Doi et al., 2010). There have been improvements in isolation of ESBL-resistant enterobacteria in food-producing animals worldwide (Geser et al., 2011). Zoonotic transmission of ESBL-producing enterobacteria has been reported (Ewers et al., 2012; Schmeidel et al., 2014). With the current global target of "one world, one health", efforts are being intensified to detect sources of antibacterial resistance (especially $\beta$ lactam resistance) in order to curb its spread in both humans and animals (Wall, 2014). Surveillance studies to assess foodproducing animals as potential reservoirs and disseminators of ESBL-resistant bacteria have been conducted in several countries in Europe (Aestrup et al., 2006; Meunier et al., 2006; Cloeckaert et al., 2007; Girlich et al., 2007; Smet et al., 2008; Wu et al., 2008; Cortes et al., 2010; Goncalves et al., 2010; Geser et al., 2011; Geser et al. 2012; Hammerrum et al., 2014), Asia (Duan et al., 2006; Tian et al., 2009; Yuan et al., 2009; Li et al., 2014; Wang et al., 2014), Africa (Jouini et al., 2007; Ben Sallem et al., 2012), North America (Wittum et al., 2010) and South America (Fernandes et al., 2009). Unfortunately, there is paucity of information on ESBLresistant bacteria in food-producing animals in Nigeria. In available literature, there are four reports (Chah and Oboegbulem, 2007; Eze et al., 2013; Duru et al. 2013 and
Inwerezua et al., 2014) on surveillance of food-producing animals as reservoirs of ESBL-resistant enterobacteria. Chah and Oboegbulem (2007), Eze et al. (2013) and Duru et al. (2013) detected $9.4 \%$ among 172 ampicillin-resistant $E$. coli, $25.5 \%$ and $22.2 \%$ among $141 \mathrm{E}$. coli isolates from chicken in Enugu, Ebonyi and Imo States in Southeast, Nigeria, respectively, whereas Inwerezua et al. (2014) detected no ESBL-resistant isolate in healthy cattle in Oyo State Southwestern, Nigeria. No study has been conducted to assess pigs reared and slaughtered in Enugu State Southeast, Nigeria, as potential reservoirs of ESBL-resistant organisms, whereas pigs constitute a major source of animal protein (because it is relatively cheaper than beef, chicken, chevron etc.) for the populace of Enugu State, Nigeria. These pigs may harbor ESBL-resistant enterobacteria which are consumed together with the pork meat by the population, hence increasing the dissemination of antimicrobial resistance genes. Therefore, there is need to assess pigs reared and slaughtered in Nigeria as reservoirs of ESBL-resistant organisms. More importantly, determination of antibiogram of isolates from foodproducing animals is essential for monitoring the spread of resistance in food-borne bacteria. This would help in evaluation of trends and identification of mitigation strategies. The objective of this study, therefore, was to isolate ESBL-resistant enterobacteria from pigs reared in Enugu State, Southeast, Nigeria, and to determine the antibacterial resistance profile of the isolates.

\section{Materials and Methods}

\section{Sampling}

This cross-sectional study was conducted between January and June 2013. Out of the 17 Local Government Areas (LGAs) in Enugu State, six were selected purposively for the study: Igbo-Eze North, Nsukka, Udenu, Udi, Eziagu and Enugu North. In these LGAs, 16 pig farms were visited. Rectal swabs were collected using sterile swab sticks from 190 randomly selected pigs reared in the farms. Each Local Government Area was visited once to avoid the possibility of re-sampling. The samples were transported aseptically in ice packs and processed within 2 hours of collection in the Veterinary Microbiology Laboratory, Department of Veterinary Pathology and Microbiology, University of Nigeria, Nsukka.

Isolation and generic identification of ESBLs-resistant enterobacteria from pigs

The swabs were cultured on Mac Conkey agar supplemented with $2 \mu \mathrm{g} / \mathrm{ml}$ of cefotaxime and incubated at $37^{\circ} \mathrm{C}$ for 24 hours aerobically. The morphology of different colonial types were described and recorded appropriately. Purification of the isolates was done by sub-culturing on plain Mac Conkey agar and incubated at $37^{\circ} \mathrm{C}$ for 24 hours. Pure cultures of the isolates were then inoculated onto nutrient agar slants, incubated at $37^{\circ} \mathrm{C}$ for 24 hours and stored in refrigerator at $4^{\circ} \mathrm{C}$ as stock cultures until needed for further analysis. Phenotypic characterization of the isolates to generic level was done by subjecting them to various tests such as Gram staining, catalase, urease, oxidase, indole, methyl red, triple sugar iron agar and citrate test, and sub-culturing on eosin methylene blue following standard methods. 


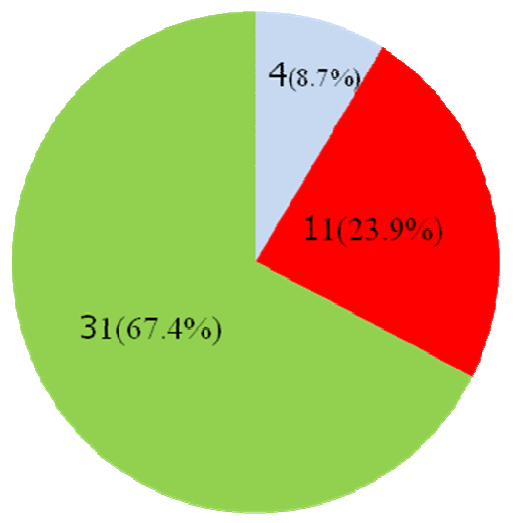

Escherichiacoli

a Salmonella

Klebsiclla

Fig. 1. Prelevance of extended-spectrum B-lactam-resistant enterobacterial isolates from pigs

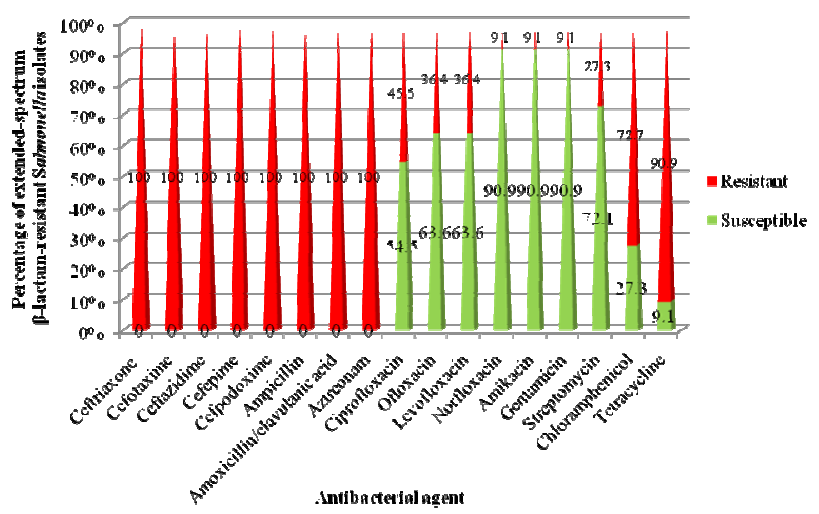

Fig. 2. Antibiogram of extended-spectrum B-lactam-resistant Salmonella isolates from pigs

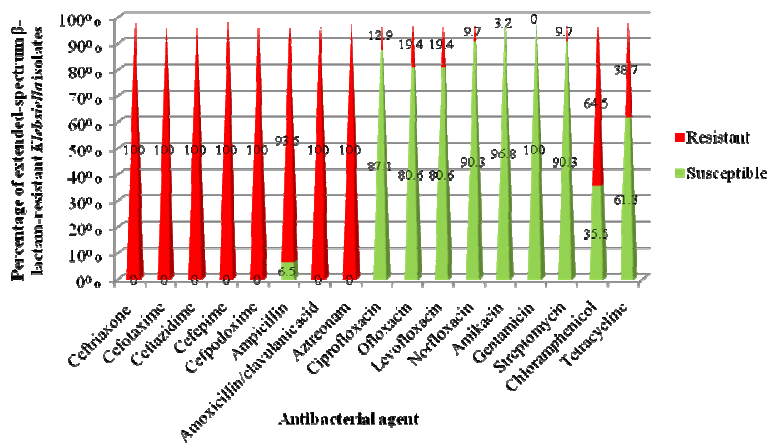

Fig. 3. Antibiogram of extended-spectrum B-lactam-resistant Klebsiella isolates from pigs

Determination of the antibiogram of the ESBLresistant enterobacterial isolates from pigs

Antibacterial susceptibility of the ESBL-resistant isolates was determined by the disc diffusion method (CLSI, 2012). The isolates were sub-cultured on nutrient agar, incubated at $37^{\circ} \mathrm{C}$ for 24 hours. Then colonies of each of the isolate were adjusted to 0.5 McFarland's turbidity standard (equivalent to $1 \times 10^{8}$ colony forming

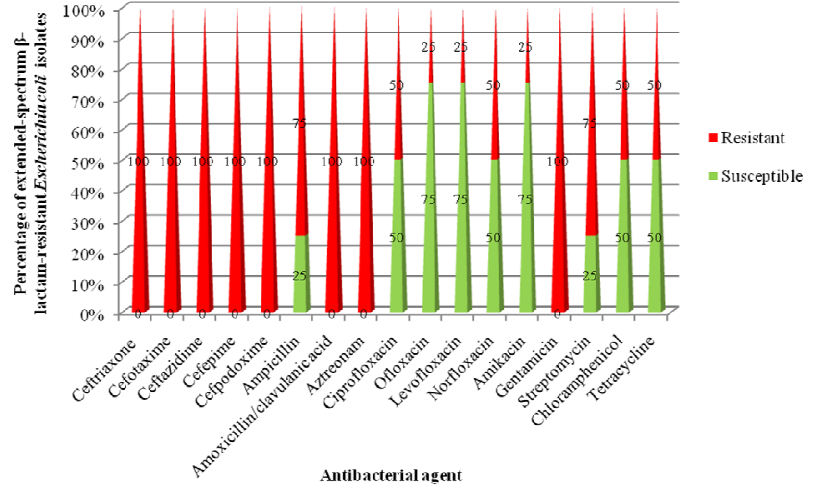

Fig. 4. Antibiogram of extended-spectrum B-lactam-resistant Escherichia coli isolates from pigs

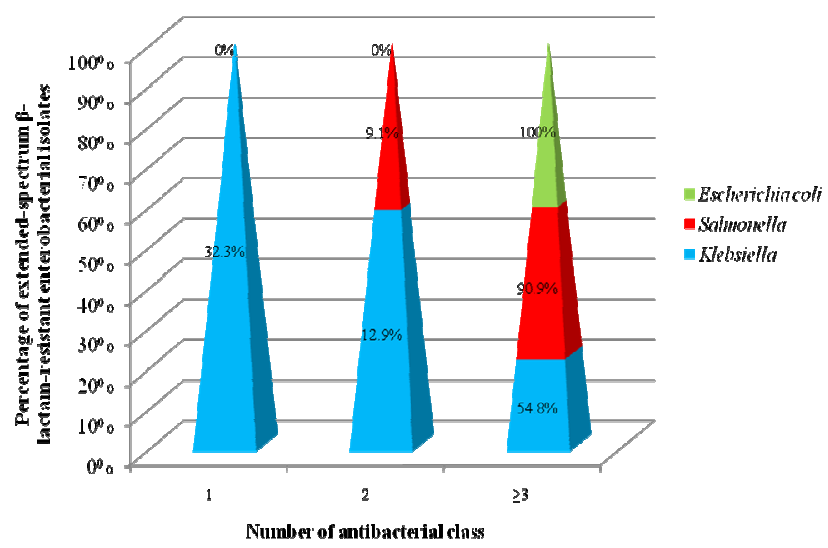

Fig. 5. Resistance of extended-spectrum B-lactam-resistant enterobacterial isolates to antibacterial classes

unit $/ \mathrm{ml}$ ) in sterile nutrient broth. The standardized broth cultures were incubated for 10 minutes at $37^{\circ} \mathrm{C}$ and then inoculated onto sterile Mueller-Hinton agar plates using sterile swab stick. Seventeen antibacterial agents (Oxoid) belonging to 5 antibacterial classes were used and they included: cefepime $(30 \mu \mathrm{g})$, cefotaxime $(30 \mu \mathrm{g})$, cefpodoxime $(10 \mu \mathrm{g})$, ceftazidime $(30 \mu \mathrm{g})$, ceftriaxone $(30 \mu \mathrm{g})$, norfloxacin $(10 \mu \mathrm{g})$, ofloxacin $(5 \mu \mathrm{g})$, levofloxacin $(5 \mu \mathrm{g})$, ciprofloxacin (5 $\mu \mathrm{g})$, streptomycin $(10 \mu \mathrm{g})$, tetracycline $(30 \mu \mathrm{g})$, gentamicin $(10 \mu \mathrm{g})$, amikacin (30 $\mu \mathrm{g})$, chloramphenicol (30 $\mu \mathrm{g})$, amoxicillin/clavulanic acid $(20 / 10 \mu \mathrm{g})$, aztreonam $(30 \mu \mathrm{g})$ and ampicillin $(10 \mu \mathrm{g})$. The discs were placed strategically on the inoculated Mueller-Hinton agar plate. The plates were incubated at $37^{\circ} \mathrm{C}$ for 24 hours. After incubation, the zone of inhibition around each disc was measured with a meter rule. Each test was performed in triplicate and the mean inhibitory zone diameter (IZD) calculated to the nearest whole millimeters for each isolate and each antibacterial agent. The IZD was interpreted as susceptible, intermediate or resistant according to the Clinical and Laboratory Standards Institute (CLSI) (2012) criteria for aerobic isolates. For the purpose of the study, isolates with intermediate-susceptibility were classified as resistant. 


\section{Results}

Prevalence of generic ESBL-resistant enterobacterial isolates from pigs

Out of the total of 190 rectal swab samples processed for isolation of ESBL-resistant enterobacteria, 46 (24.2\%) gave positive culture. Of the $46 \mathrm{ESBL}$-resistant isolates, 4 (8.7\%) were E. coli, 11 (23.9\%) were Salmonella species, while 31 (67.4\%) were Klebsiella species (Fig. 1).

Out of the 11 ESBL-resistant Salmonella isolates, all (100\%) were resistant to ceftriaxone, cefotaxime, ceftazidime, cefepime, cefpodoxime, ampicillin, amoxicillin/clavulanic acid and aztreonam, $5(45.5 \%)$ to ciprofloxacin, $4(36.4 \%)$ to ofloxacin and levofloxacin, 1 (9.1\%) to norfloxacin, amikacin and gentamicin, 3 (27.3\%) to streptomycin, $8(72.7 \%)$ to chloramphenicol and 10 (90.9\%) to tetracycline (Fig. 2).

Out of the 31 ESBL-resistant Klebsiella isolates, all (100\%) were resistant to ceftriaxone, cefotaxime, ceftazidime, cefepime, cefpodoxime, amoxicillin/clavulanic acid and aztreonam, $29(93.5 \%)$ to ampicillin, $4(12.9 \%)$ to ciprofloxacin, $6(19.4 \%)$ to ofloxacin and levofloxacin, 3 (9.7\%) to norfloxacin and streptomycin, $20(64.5 \%)$ to chloramphenicol and $12(38.7 \%)$ to tetracycline (Fig. 3). None (0\%) of the Klebsiella isolates was resistant to gentamicin.

Out of the 4 ESBL-resistant E. coli isolates, all (100\%) were resistant to ceftriaxone, cefotaxime, ceftazidime, cefepime, cefpodoxime, amoxicillin/clavulanic acid, aztreonam and gentamicin, $3(75 \%)$ to ampicillin and streptomycin, $2(50 \%)$ to ciprofloxacin, norfloxacin, chloramphenicol and tetracycline and 1 (25\%) to ofloxacin, levofloxacin and amikacin (Fig. 4).

Out of the 31 Klebsiella isolates, 10 (32.3\%) were resistant to one class of the antibacterial agents tested, 4 $(12.9 \%)$ to two classes, while $17(54.8 \%)$ were resistant to three or more of the antibacterial agents tested (Fig. 5). Of the 11 Salmonella isolates, 1 (9.1\%) was resistant to two antibacterial classes tested, while 10 (90.9\%) were resistant to three or more classes. All $(100 \%)$ the $E$. coli isolates were resistant to three or more classes of the antibacterial agents tested.

\section{Discussion}

In this study, the prevalence and antibiogram of ESBLresistant enterobacterial isolates from pigs reared in Enugu State, Nigeria, was determined. The fact that $46(24.2 \%)$ enterobacteria isolates were obtained from the cultured rectal swab samples using Mac Conkey agar supplemented with $2 \mu \mathrm{g} / \mathrm{ml}$ of cefotaxime, suggested that the isolates were ESBL-resistant enterobacteria. Cefotaxime is a 3rdgeneration cephalosporin (extended-spectrum $\beta$-lactam) and its resistance is mediated by ESBLs (Geser et al., 2012). Therefore, the growth of the isolates on the extendedspectrum $\beta$-lactam-supplemented medium suggested that they might have produced ESBLs. ESBL-resistant enterobacteria isolation prevalence of $24.2 \%$ recorded in this study is higher than $8.4 \%$ prevalence of ESBLproducing Enterobacteriaceae reported by Reist et al. (2013) in calves slaughtered in Switzerland. Isolation of three genera (Klebsiella, E. coli and Salmonella) of ESBL-resistant enterobacteria in this study, suggested that there could have been acquisition/transfer of genes encoding for ESBLs production by/among the enterobacterial organisms (Schmeidel et al., 2014). The $67.4 \%$ Klebsiella isolation prevalence suggested that the genus may have the capacity of acquiring genes encoding for ESBLs more than Salmonella and $E$. coli with isolation prevalence of $23.9 \%$ and $8.7 \%$, respectively. Among ESBL-resistant enterobacteria, Klebsiella acquired the greatest variety of genes encoding for ESBLs production and it produced the greatest variety of ESBLs (Paterson et al., 2003; Nobrega et al., 2013). Studies conducted elsewhere in Africa also reported isolation of ESBL-resistant Klebsiella more frequently than any other genus in Enterobacteriaceae (Blomberg et al., 2005; Gangoue-Pieboji et al., 2005). The $67.4 \%$ isolation prevalence of ESBL-resistant Klebsiella in the present study is higher than $28.6 \%$ isolation prevalence of ESBLproducing Klebsiella reported by Guandong and Avci (2013) in food of animal origin in Turkey.

Salmonella was isolated in this study at the prevalence of 23.9\%. Isolation of ESBL-resistant Salmonella from foodproducing animals have been reported in Brazil (Fernandes et al., 2009), Germany (Rodriguez et al., 2009) and from a slaughterhouse and poultry farm in Korea (Bae et al., 2013) and Spain (Silva et al., 2013), respectively.

In the current study, isolation prevalence of ESBLresistant $E$. coli was $8.7 \%$. This result is lower than $9.4 \%$ isolation prevalence of ESBLs-producing $E$. coli reported by Chah and Oboegbulem (2007) among 171 ampicillinresistant $E$. coli isolates from chicken in Nigeria. Smet et al. (2008), Geser et al. (2011), Garcia-Graells et al. (2010) and Wasinski et al. (2013) reported 60, 92.3, 42 and 11.7\% isolation prevalence of ESBLs-producing $E$. coli in poultry carcasses in Belgium, broilers in Belgium, pigs and cattle in Switzerland, and meat samples in Poland, respectively. In the Netherlands, Overdevest et al. (2011) and Leversteinvan Hall et al. (2011) reported 80 and 94\% prevalence of ESBLs-producing $E$. coli in chicken meat and raw meat, respectively. Escudero et al. (2010), Schmid et al. (2013), Adenaike et al. (2013), Ben Sallem et al. (2012) and Guandong and Avci (2013) reported 72, 21.4, 45, 13.8 and $44.4 \%$ prevalence of ESBL-producing $E$. coli from pigs in Spain, beef cattle in Germany, roasted beef in Nigeria, foodproducing animals in Tunisia and food of animal origin in Turkey, respectively. The isolation prevalence of ESBLresistant $E$. coli $(8.7 \%)$ in this study is lower when compared with those of the previous studies. The variation in isolation prevalence of ESBL-resistant enterobacteria recorded in these studies could be related to differences in the use of $\beta$ lactams in human and veterinary medicine in the various study areas. The findings of this study therefore suggest sizeable prevalence of ESBL-resistant enterobacteria in pigs reared in Enugu State, Nigeria. This portends huge adverse health impact on the food chain in the study area.

In the disc synergy/combination test for phenotypic detection of ESBLs production, ceftazidime and cefotaxime plus clavulanic acid (clavulanate) discs were used (Warren $e t$ al., 2001; CLSI, 2012). Resistance of the isolates to the drugs was tested in the hereby study (with single discs) to further confirm that they produced ESBLs. The fact that all 
276

tested isolates were resistant to the extended-spectrum $\beta$ lactams tested (e.g. cefepime, cefpodoxime, ceftriaxone, ceftazidime and cefotaxime) further suggested that they produced extended-spectrum $\beta$-lactamases (ESBLs). ESBLs are hydrolytic enzymes that breakdown the $\beta$-lactam ring of $\beta$-lactams, extended-spectrum $\beta$-lactams $\left(3^{\text {rd }}\right.$ and $4^{\text {th }}$ generation cephalosporins/ $\beta$-lactams) and monobactams (Garcia-Graells et al., 2010). The 100\% resistance to ESBL in this study is similar to the findings of Duru et al. (2013) among ESBLs-producing $E$. coli isolates from poultry in Imo State, Nigeria. The $100 \%$ cefotaxime resistance observed in this study is higher than $84.6 \%$ cefotaxime resistance reported by Schmid et al. (2013) among ESBLsproducing E. coli isolates from beef cattle in Germany. High rates $(100 \%)$ of resistance to ampicillin and aztreonam among all the genera of enterobacterial isolates were observed in the hereby experiment, further suggesting the production of ESBLs by the isolates. These drugs (ampicillin - $\beta$-lactam and aztreonam - monobactam) are $\beta$-lactams which are rendered ineffective by ESBLs (GarciaGraells et al., 2010). Ampicillin resistance rate (100\%) recorded in this study is similar to the findings of Guandong and Avci (2013), who reported 100\% ampicillin resistance among ESBLs-producing Klebsiella and E. coli isolates from food of animal origin in Turkey. The recorded 100\% ampicillin resistance in this study is higher than $75 \%$ ampicillin resistance reported among ESBLs-producing $E$. coli isolates from roasted beef in Nigeria (Adenaike et al., 2013). Rate of resistance (100\%) to aztreonam by all the genera in the present study is higher when compared with 65.4 and $28.9 \%$ aztreonam resistance reported by Schmid $e t$ al. (2013) and Guandong and Avci (2013) among ESBLresistant $E$. coli isolates from beef cattle in Germany and food of animal origin in Turkey, respectively. It is also higher than $42.9 \%$ aztreonam resistance reported by Guandong and Avci (2013) among ESBLs-producing Klebsiella isolates in Turkey.

High rate $(100 \%)$ of amoxicillin/clavulanic acid by all the genera in this study suggestsed that the isolates might have produced other $\beta$-lactamases such as AmpC cephalosporinases. AmpC cephalosporinases mediate resistance to $3^{\text {rd }}$-generation $\beta$-lactams/cephalosporins, but are not inhibited by clavulanic acid and other $\beta$-lactamase inhibitors (such as sulbactam and tazobactam) (Ben Sallem et al., 2012). Conversely, the ESBLs are inhibited by clavulanic acid and other $\beta$-lactamase inhibitors (GarciaGraells et al., 2010; Ben Sallem et al., 2012). Amoxicillin/clavulanic acid resistance rate (100\%) in this study is higher when compared with 53.8, 6.7 and $90 \%$ amoxicillin/clavulanic acid resistance reported by Schmid $e t$ al. (2013), Guandong and Avci (2013) and Duru et al. (2013) among ESBL-producing E. coli isolates from cattle in Germany, food of animal origin in Turkey and poultry in Nigeria, respectively. It is also higher than $3.1 \%$ amoxicillin/clavulanic acid resistance among ESBLsproducing Klebsiella isolates reported by Guandong and Avci (2013). The high rate (100\%) of resistance to all the tested $\beta$ lactams demonstrated by all the genera in this study, may have resulted due to the frequent use of $\beta$-lactams in preparation of animal drugs and treatment of bacterial infections in animals within the study area (Chah and
Nweze, 2001). Recently, the use of extended-spectrum $\beta$ lactams in humans and animals has tremendously increased in Nigeria. This could have resulted in the high resistance to all the $\beta$-lactams tested in this study. The Salmonella isolates exhibited the highest rate of resistance to the $\beta$-lactams, which further suggested that isolates in the genus may have been exposed more to the drugs than their counterparts in the other genera obtained in this study. The high rate of resistance to ESBL in this study calls for real concern, as these drugs (ESBL) are the only available drugs for treatment of resistant bacteria. If the resistance continues to spread at the rate observed in this study, carbapenems may be resorted for use in production of food animals, while this situation could result in development of superbugs.

In this study, resistance rates of the Salmonella isolates to ciprofloxacin (45.5\%), ofloxacin and levofloxacin (36.4\%), and norfloxacin $(9.1 \%)$, suggested that isolates in the genus have not developed complete resistance to fluoroquinolones. This may also explain similar low resistance rate of the Klebsiella isolates to fluoroquinolones. The low fluoroquinolone resistance among the Salmonella and Klebsiella isolates may be due to infrequent use of these drugs in swine medicine within the study area. Nevertheless, the low fluoroquinolone resistance may not be unconnected to the ESBLs which the isolates might have produced (Morosini et al., 2006). Cross-resistance to fluoroquinolone by ESBL-resistant Salmonella isolates has been reported (Pasquali et al., 2005). High resistance (50\%) of the E. coli isolates to ciprofloxacin and norfloxacin in this study suggested that they exerted higher selection against the drugs than to the other tested fluorquinolones with lower rates of resistance (25\% for ofloxacin and levofloxacin). Geser et al. (2011), Schmid et al. (2013), and Guandong and Avci (2013) reported 42.9, 15.4 and 31.1\% ciprofloxacin resistance among ESBLs-producing $E$. coli isolates from pigs and cattle in Switzerland, beef cattle in Germany and food of animal origin in Turkey, respectively. The rates of ciprofloxacin resistance in these previous studies are lower when compared with the ciprofloxacin resistance (50\%) among $E$. coli isolates in the present study. However, the result is lower than $78 \%$ ciprofloxacin resistance reported by Eze et al. (2013) among ESBLsproducing E. coli isolates from chicken in Nigeria. The $25 \%$ ofloxacin resistance rate in this study is lower when compared with 68.8 and $69.4 \%$ reported by Li et al. (2014) and Eze et al. (2013) among ESBLs-producing E. coli isolates from chicken in China and Nigeria, respectively. The $12.9 \%$ ciprofloxacin resistance shown by Klebsiella isolates in this study contrasts $23.8 \%$ ciprofloxacin resistance exhibited by ESBLs-producing Klebsiella isolates from food of animal origin in Turkey (Guandong and Avci, 2013). Differences in fluoroquinolones resistance in this study may suggest variation in usage of the drugs in production of food animals in the study areas. Eze et al. (2013) noted that the use of fluoroquinolones in foodproducing animals is not common in Nigeria, so this may account for the relative lower fluoroquinolone resistance rates observed in this study. Nevertheless, cross resistance of ESBLs-resistant Enterobacteriaceae to fluoroquinolones have severally been reported (Jacoby, 2005; Frank et al., 2011). 
Low rates of resistance to amikacin and gentamicin (9.1\%), and streptomycin (27.3\%) by the Salmonella isolates suggested that they exerted low pressure for selection against the aminoglycosides. Similar low resistance rates were exhibited by the Klebsiella isolates against streptomycin (9.7\%), amikacin (3.2\%) and gentamicin $(0 \%)$. The high resistance rates (100\% for gentamicin, $75 \%$ for streptomycin and amikacin) among the $E$. coli isolates suggested that they exerted higher selection pressure against the aminoglycosides, more than their counterparts in the other genera. The high streptomycin resistance may be related to the fact that streptomycin is frequently used in combination with penicillin to exert a broad spectrum action for treatment of bacterial infections of pigs in the study area. The $75 \%$ streptomycin resistance in this study is similar to $75 \%$ streptomycin resistance recorded among ESBLs-producing E. coli isolates from pigs and cattle in Switzerland (Geser et al., 2012). It is higher when compared with $60 \%$ streptomycin resistance reported by Reich et al. (2010) among ESBLs-producing E. coli isolates from chicken carcasses in Germany. The 100\% gentamicin resistance among the $E$. coli isolates in this study is higher when compared with 42.9, 15.4, 6.7 and $34.5 \%$ gentamicin resistance among ESBLs-producing $E$. coli isolates reported by Geser $e$ al. (2011) in Switzerland, Schmid et al. (2013) in Germany, Guandong and Avci (2013) in Turkey and Li et al. (2014) in China, respectively. Rate of amikacin resistance (75\%) among the E. coli isolates in this study, contrast with $8.4 \%$ amikacin resistance reported by Li $e t a l$. (2014) among ESBLs-producing E. coli isolates from chicken in China. Low amikacin resistance among the Salmonella (9.1\%) and Klebsiella (3.2\%) isolates suggested that these genera exerted low pressure for selection against the drug. In Turkey, an amikacin resistance rate of $4.4 \%$ was reported by Guandong and Avci (2013). These results are higher than the amikacin resistance among Klebsiella isolates in this study, but it is lower when compared with 9.1\% amikacin resistance among the Salmonella isolates obtained in the present study. The aminoglycoside resistance observed in this study is probably due to production of ESBLs by the isolates (Carattoli, 2008). Cross-resistance of ESBL-resistant enterobacteria to aminoglycosides has also been reported (Morosini et al., 2006).

The high rate (72.7\%) of chloramphenicol resistance by the Salmonella isolates suggested that the genus have acquired resistance against the drug more than the E. coli (50\%) and Klebsiella isolates (64.5\%). Chloramphenicol resistance rates recorded in this study is higher than the findings of Reich et al. (2010) and Geser et al. (2011) who reported 38 and $47.6 \%$ chloramphenicol resistance among ESBLs-producing E. coli isolates in Switzerland and Germany, respectively. High rate (90.9\%) of tetracycline resistance among the Salmonella isolates suggested that they exerted high selection pressure for resistance against the drug more than the Klebsiella (38.7\%) and E. coli (50\%) isolates. This demonstrable resistance to tetracycline by the enterobacterial isolates may have resulted due to frequent use of the drug in treatment of bacterial diseases in pigs within the study area. Tetracycline is a broad-spectrum antibacterial agent often used for prophylaxis and treatment of infections in food-producing animals. Geser et al. (2011), Adenaike et al. (2013), Guandong and Avci (2013) and Reich et al. (2010) reported 71.4, 65, 77.8 and more than $50 \%$ tetracycline resistance among ESBLs-producing E. coli isolates from pigs in Switzerland, cattle in Nigeria, food of animal origin in Turkey and chicken carcasses in Germany, respectively. The tetracycline resistance rates reported in the previous studies is higher when compared with the recorded $50 \%$ tetracycline resistance among the $E$. coli isolates in the present study. Guandong and Avci (2013) reported 69.8\% tetracycline resistance among ESBL-producing Klebsiella isolates in Turkey. This result is higher when compared with $38.7 \%$ tetracycline resistance exhibited by Klebsiella isolates in this study. ESBLs-resistant enterobacterial isolates have been reported to exhibit co-resistance to tetracycline and phenicols (Morosini et al., 2006).

Multidrug resistance implies resistance to three or more classes of antibacterial agent (Tenover et al., 2006). Resistance of all the E. coli isolates to three or more of the antibacterial agents suggested that isolates in the genus exhibited higher multidrug resistance when compared with the multidrug resistance of the Salmonella (90.9\%) and Klebsiella (54.8\%) isolates. Adenaike et al. (2013) reported $89 \%$ multidrug resistance among ESBL-resistant E. coli isolates from roasted beef in Northern Nigeria. Studies showed that ESBLs-producing enterobacteria are multidrug resistant (Guandong and Avci, 2013; Blaak et al., 2014). Therefore, the multidrug resistance observed in this study may be as a result of ESBLs that the isolates might have produced (Blaak et al., 2014). The high multidrug resistance exhibited among enterobacterial isolates in this study, portends adverse impact on the food chain in the study area. This is because consumers of pork meat and associated processed foods from these pigs could acquire these multidrug resistant organisms. Consequently, there could be compromise in antibacterial therapy in colonized and/or infected individuals (Garcia-Graells et al., 2010).

\section{Conclusions}

This study has shown that ESBL-resistant enterobacteria are harboured by a sizeable percentage (24.2\%) of pigs reared in Enugu State, Southeastern Nigeria. Klebsiella is the most prevalent genus of ESBLresistant enterobacteria colonizing pigs reared within the study area. Thus, pigs reared in the study area are potential reservoirs and disseminators of ESBL-resistant enterobacteria and genes encoding for ESBLs production. This has tremendous impact on the food chain and ecology of antibacterial resistance. However, further studies to elucidate the types of ESBLs genes harbored by the isolates are recommended.

\section{References}

Aarestrup FM, Hasman H, Agerso Y, Jensen LB, Harksen S, Svensmark B (2006). First description of blaCTX-M-1-carrying Escherichia coli isolates in Danish primary food production. Journal of Antimicrobial Chemotherapy 57:1258-1259.

Adenaike O, Olonitola OS, Ameh JB, Whong CMZ (2013). Incidence 
of extended spectrum $\beta$-lactamase producing bacteria and multidrug resistance strains from processed meat 'suya' sold in a university community. The International Journal of Engineering and Science 2(12):1-6.

Bae DH, Dessie HK, Baek HJ, Kim SG, Lee HS, Lee YJ (2013). Prevalence and characteristics of Salmonella spp. isolated from poultry slaughterhouses in Korea. Journal of Veterinary Medical Science 75(9):1193-1200.

Ben Sallem R, Ben Slama K, Saenz Y, Rojo-Bezares B, Estepa V, Jouini A, Gharsa H, Klibi N, Boudaous A, Torres C (2012). Prevalence and characterization of extended-spectrum beta-lactamase (ESBL)and CMY-2-producing Escherichia coli isolates from healthy foodproducing animals in Tunisia. Foodborne Pathogens and Disease 9(12):1137-1142.

Blaak H, Hamidjaja RA, van Hoek AHAM, de Heer L, Husman AM, Schets FM (2014). Detection of extended-spectrum beta-lactamase (ESBL)-producing Escherichia coli on flies at poultry farms. Applied and Environmental Microbiology 80(1):239-246.

Blomberg B, Jureen R, Manji KP (2005). High rate of fatal cases of pediatric septicemia caused by Gram-negative bacteria with extended spectrum $\beta$-lactamases in Dar es Salaam, Tanzania. Journal of Clinical Microbiology 43:745-749.

Canton R, Akova M, Carmeli Y, Giske CG, Glupczynski Y, Gniadkowski M, Livermore DM, Miriagou V, Nass T, Rossolini GM, Samuelsen O, Seifert h, Woodford N, Nordmann P (2012). Rapid evolution and spread of carbapenemases among Enterobacteriaceae in Europe. Clinical Microbiology and Infection $18(5): 413-431$.

Carattoli A (2008). Animal reservoirs for extended-spectrum betalactamase producers. Clinical Microbiology and Infection 14:117123.

Chah KF, Oboegbulem SI (2007). Extended spectrum beta-lactamase production among ampicillin-resistant Esherichia coli strains from chicken in Enugu State, Nigeria. Brazilian Journal of Microbiology 38:681-686.

Clinical and Laboratory Standards Institute (CLSI) (2012). Performance standards for antimicrobial susceptibility testing; twenty-second informational supplement, M100-S2232(3):62-78.

Cloeckaert A, Praud K, Doublet B, Bertini A, Carattoli A, Butaye P, Imberechts H, Bertrand S, Collard J-M, Arlet G, Weill F-X (2007). Dissemination of an extended-spectrum $\beta$-lactamase blaTEM-52 gene-carrying IncI1 plasmid in various Salmonella enterica serovars isolated from poultry and humans in Belgium and France between 2001 and 2005. Antimicrobial Agents and Chemotherapy 51:18721875.

Cortes P, Blance V, Morca A, Dahbi G, Blanco JE, Blanco M, Lopez C, Andreu A, Navarro F, Alonso MP, Bou G, Blanco J, Llagostera M (2010). Isolation and characterization of potentially pathogenic antimicrobial-resistant Escherichia coli strains from chicken and pig farms in Spain. Applied and Environmental Microbiology 76:27992805.

Doi Y, Paterson DL, Egea P, PascuaL A, Lopez-Cerero L, Navarro MD, Adams-Haduch JM, Qureshi ZH, Sidjabat HE, Rodriguez-Bano J (2010). Extended-spectrum and CMY-type beta-lactamase- producing Escherichia coli in clinical samples and retail meat from Pittsburgh, USA and Seville, Spain. Clinical Microbiology and Infection 16:33-38.

Duan RS, Sit TH, Wong SS, Wong RC, Chow KH, Mak GC, Yam WC, Ng LT, Yuen KY, Ho PL (2006). Escherichia coli producing CTX-M $\beta$-lactamases in food animals in Hong Kong. Microbial Drug Resistance 12:145-148.

Duru C, Nwanegbo E, Adikwu M, Ejikeugwu C, Esimone C (2013). Extended-spectrum beta-lactamase-producing Escherichia coli strains of poultry origin in Owerri, Nigeria. World Journal of Medical Sciences 8(4):349-354.

Escudero E, Vinue L, Teshager T, Torres C, Moreno MA (2010). Resistance mechanisms and farm-level distribution of fecal Escherichia coli isolates resistant to extended-spectrum cephalosporins in pigs in Spain. Research in Veterinary Science 88:83-87.

Ewers C, Bethe A, Semmler T, GuentherS, Wieler LH(2012). Extendedspectrum $\beta$-lactamase-producing and AmpC-producing Escherichia coli from livestock and companion animals, and their putative impact on public health: a global perspective. Clinical Microbiology and Infection 18:646-655.

Eze E, Nwakeze E, Oji A, Ejikeugwu C, Iroha I (2013). Microbiological investigation of Escherichia coli isolates from cloacal and feacal swabs of broiler chickens for extended- spectrum beta-lactamase (ESBL) enzymes. Journal of Pharmacy and Biological Sciences 7(5):96-99.

Fernandes SA, Paterson DL, Ghilardi-Rodrigues AC, Adams-Haduch JM, Tavechio AT, Doi Y (2009). CTX-M-2-producing Salmonella Typhimurium isolated from pediatric patients and poultry in Brazil. Microbial Drug Resistance 15(4):317-321.

Frank T, Mbecko JR, Misatou P, Monchy D (2011). Emergence of quinolone resistance among extended-spectrum beta-lactamaseproducing Enterobacteriaceae in the Central African Republic: genetic characterization. BioMed Central Research Notes 4:309.

Garcia-Graells C, Bottleldoorn N, Dierick K (2014). Microbial surveillance of ESBL E.coli in poultry meat, a possible vehicle for transfer of antimicrobial resistance to humans. Retrived 2014 October 27 from https://www.wivisp.be/Documents/Scientific\%20brochure\%20123/ISP THEME2 ARTICLE Microbial\%20Surveillance\%20of\%20E.\%20coli\%20i n\%20poultry\%20meat.pdf.

Gangoue-Pieboji J, Bedenic B, Koulla-Shiro S (2005). Extendedspectrum $\beta$-lactamase producing Enterobacteriaceae in Yaounde, Cameroon. Journal of Clinical Microbiology 43:3273-3277.

Geser N, Stephan R, Hachler H (2012). Occurrence and characteristics of extended-spectrum $\beta$-lactamase (ESBL) producing enterobacteriaceae in food producing animals, minced meat and raw milk. BMC Veterinary Research 8:21.

Geser N, Stephan R, Kuhnert P, Zbinden R, Kaeppeli U, Cernela N, Haechler H (2011). Fecal carriage of extended-spectrum betalactamase-producing enterobacteriaceae in swine and cattle at slaughter in Switzerland. Journal of Food Protection 74(3):446449.

Girlich D, Poirel L, Carattoli A, Kempf I, Lartigue MF, Bertini A, Nordmann P (2007). Extended-spectrum (beta)-lactamase CTXM-1 in Escherichia coli in healthy poultry in France. Applied and Environmental Microbiology 73:4681-4685. 
Gniadkowski M (2001). Evolution and epidemiology of extendedspectrum beta-lactamases (ESBLs) and ESBL-producing microorganisms. Clinical Microbiology and Infection 7:597-608.

Goncalves A, Silva TC, Carneiro C, Radhouani H, Coelho C, Aruajo Rodrigues J, Vinue L, Somalo S, Poeta P, Igrejas G (2010). Genetic characterization of extended-spectrum $\beta$-lactamases in Escherichia coli isolates of pigs from a Portuguese intensive swine farm. Foodborne Pathogens and Disease 7:1569-1573.

Guandogan N, Avci E (2013). Prevalence and antibiotic resistance of extended-spectrum beta-lactamase (ESBL) producing Escherichia coli and Klebsiella species isolated from foods of animal origin in Turkey. Africal Journal of Microbiology Research 7(31):40594064.

Hammad AM, Ahmed AM, Ishida Y, Shimamoto T (2008). First characterization and emergence of SHV-60 in raw milk of a healthy cow in Japan. Journal of Veterinary Medical Science 70:1269-1272.

Hammerum AM, Larsen J, Andersen VD, Lester CH, Skytte TSS, Hansen F, Olsen SS, Mordorst H, Skov RL, Aaerestrup FM, Agerso Y (2014). Characterization of extended-spectrum $\beta$ lactamase (ESBL)-producing Escherichia coli obtained from Danish pigs, pig farmers and their families from farms with high or no consumption of third- or fourth-generation cephalosporins. Journal of Antimicrobial Chemotherapy 69(10):2650-2657.

Inwezerua C, Mendonça N, Calhau V, Domingues S, Adeleke OE, Da Silva GJ (2014). Occurrence of extended-spectrum beta-lactamases in human and bovine isolates of Escherichia coli from Oyo state, Nigeria. The Journal of Infection in Developing Countries $8(6): 774779$.

Jacoby GA (2012). Mechanism of resistance to quinolones. Clin Infect Dis 41 (Supplement 2):S120-S126.doi:10.1086/428052.

Jensen LB, Hasman H, Agerso Y, Emborg HD, Aarestrup FM (2006). First description of an oxyimino-cephalosporin-resistant, ESBLcarrying Escherichia coli isolated from meat sold in Denmark. Journal of Antimicrobial Chemotherapy 57:793-794.

Jouini A, Vinue L, Ben Slama K, Saenz Y, Klibi N, Hammami S, Boudabous A, Torres C (2007). Characterization of CTX-M and SHV extended-spectrum beta-lactamases and associated resistance genes in Escherichia coli strains of food samples in Tunisia. Journal of Antimicrobial Chemotherapy 60:1137-1141.

Leverstein-van Hall MA, Dierikx CM, Cohen SJ, Voets GM, van den Munckhof MP, van Essen-Zandergen A, ... Zeng M (2014). Prevalence and characteristics of extended-spectrum $\beta$-Lactamase and plasmid-mediated fluoroquinolone resistance genes in Escherichia coli isolated from chickens in Anhui Province, China. PLoS One 9(8):e104356. doi:10.1371/journal.pone.0104356.

Meunier D, Jouy E, Lazizzers C, Kobisc M, Madec JY (2006). CTX-M1 - and CTX-M-15-type $\beta$-lactamases in clinical Escherichia coli isolates recovered from food-producing animals in France. International Journal of Antimicrobial Agents 28:402-407.

Morosini MI, Garcia-Castillo M, Coque TM, Valverde A, Novai SA, Loza E, Baqquero F, Canton R (2006). Antibiotic coresistance in extended-spectrum $\beta$-lactamase-producing Enterobacteriaceae and in vitro activity of tigecycline. Antimicrobial Agents and Chemotherapy 50(8):2695-2699.
Nobrega DB, Marcos VSG, Guimaraes FF, Riboli DF, Cunha MLRS, Langoni H, Pantoga JCF, Lucheis SB (2013). Molecular epidemiology and extended-spectrum $\beta$-lactamases production of Klebsiella pneumoniae isolated from three dairy herds. Pesquisa Veterinária Brasileira 33(7):855-859.

Overdevest I, Willemsen I, Rijnsburger M, Eustace A, Xu L, Hawkey P, Heck M, Savelkoul P, Vandenroucke-Grauls C, van der Zwaluw K, Huijsdens X, Kluytmans J (2011). Extended-spectrum $\beta$-lactamase genes of Escherichia coli in chicken meat and humans, The Netherlands. Emerging Infectious Diseases 17(7):1216-1222.

Pasquali F, Kehrenberg C, Manfreda G, Schwarz S (2005). Physical linkage of Tn3 and part of Tn1721 in a tetracycline and ampicillin resistance plasmid from Salmonella Typhimurium. Journal of Antimicrobial Chemotherapy 55:562-565.

Paterson DL, Hujer KM, Hujer AM, Yeiser B, Bonomo MD, Rice LB, Bonomo RA, The International Klebsiella Study Group (2003). Extended-spectrum $\beta$-lactamases in Klebsiella pneumoniae bloodstream isolates from seven countries: dominance and widespread prevalence of SHV- and CTX-M-type $\beta$-lactamases. Antimicrobial Agents and Chemotherapy 47:3554-3560.

Reich F, Atanassova V, Klein G (2010). Extended-spectrum Blactamase- and AmpC-producing enterobacteria in healthy broiler chickens, Germany. Emerging Infectious Diseases 19(8):12531259.

Reist M, Geser N, Hachler H, Scharrer S, Stephan R (2013). ESBLproducing Enterobacteriaceae: occurrence, risk factors for fecal carriage and strain traits in the Swiss slaughter cattle population younger than 2 years sampled at abattoir level. PLoS One 8(8):e71725. doi:10.1371/journal.pone.0071725.

Rodriguez I, Barownick W, Helmuth R, Mendoza MC, Rodicio MR, Schroeter A, Guerra B (2009). Extended-spectrum beta-lactamases and AmpC beta-lactamases in ceftiofur resistant Salmonella enterica isolates from food and livestock obtained in Germany during 200307. Journal of Antimicrobial Chemotherapy 64:301-309.

Schmedeil J, Falgenhauer L, Domann E, Bauerfeind R, PrengerBerninghoffE, Imirzalioglu C, Chakraorty T (2014). Multiresistant extended-spectrum $\beta$-lactamase-producing Enterobacteriaceae from humans, companion animals and horses in central Hesse, Germany. BMC Microbiology 14:187.

Schmid A, Hörmansdorfer U, Messelhausser A, Kasbohrer C, SauterLouis, Mansfeld R (2013). Prevalence of extended-spectrum $\beta$ lactamase-producing Escherichia coli on Bavarian dairy and beef cattle farms. Applied and Environmental Microbiology 79(9):3027-3032.

Silva KC, Fontes LC, Moreno AM, Astolfi-Ferreira CS, Ferreira AJ, Lincopan N (2013). Emergence of extended-spectrum betaLactamase (CTX-M-2)-producing Salmonella enterica serovars Schwarzengrund and Agona in poultry farms. Antimicrobial Agents and Chemotherapy 57(7):3458-3459.

Smet A, Martel A, Persoons D, Dewulf J, Heyndricks M, Catry B, Herman L, Haesebrouck F, Butaye P (2008). Diversity of extendedspectrum $\beta$-lactamases and class $\mathrm{C} \beta$-lactamases among cloacal Escherichia coli isolates in Belgian broiler farms. Antimicrobial Agents and Chemotherapy 52:1238-1243. 
280

Tenover FC (2006). Mechanisms of antimicrobial resistance in bacteria. American Journal of Medicine 119(6):S3-S10.

Tian GB, Wang HN, Zou LK, Tang JN, Zhao YW, My Y, Tang JY, Zhang Y, Zhang AY, Yang X, Xu CW, Fu YJ (2009). Detection of CTX-M-15, CTX-M-22, and SHV-2 extended-spectrum $\beta$ lactamases (ESBLs) in Escherichia coli fecal sample isolates from pig farms in China. Foodborne Pathogens and Disease 6:297-304.

Wall P (2014). One health and the food chain: maintaining safety in a globalised industry. Veterinary Record 174:189-192.

WangJY, Tang P, Cui EH, Wang LQ, Liu WH, Ren JJ, Wu N, YuanHao, Qiu YH, Liu HJ (2014). Characterization of antimicrobial resistance and related resistance genes in Escherichia coli strains isolated from chickens in China during 2007-2012. African Journal of Microbiology Research 7(46):5238-5247.

Warren AL, Townsend KM, King T, Moss S, O’Boyle D, Yates RM, Trott DJ (2001). Multi-drug resistant Escherichia coli with extended-spectrum $\beta$-lactamase activity and fluoroquinolone resistance isolated from clinical infections in dogs. Australian Veterinary Journal 79:621-623.

Wasinski B, Rozanska H, Osek J (2013). Occurrence of extended spectrum $\beta$-lactamase and AmpC-producing Escherichia coli in meat samples. Bulletin of the Veterinary Institute in Pulawy 57:513517.

Waters AE, Contente-Cuomo T, Buchhagen J, Liu CM, Watson L, Pearce K, Foster JT, Bowers J, Driebe EM, Engelthaler DM, Keim PS, Price LB (2011). Multidrug-resistant Staphylococcus aureus in US meat and poultry. Clinical Infectious Diseases doi: 10.1093/cid/cir181.
World Health Organization (WHO) (2014). Antimicrobial resistance global report on surveillance. Retrived 2015 January 12 from http://www.who.int/drugresistance/documents/surveillancereport /en/.

Wittum TE, Mollenkopf DF, Daniels JB, Parkinson AE, Mathews JL, Fry PR, Abley MJ, Gebreyes WA (2010). CTXM-type expendedspectrum $\beta$-lactamases present in Escherichia coli from the feces of cattle in Ohio, United States. Foodborne Pathogens and Disease 7:1575-1579.

Wu S, Chouliara E, Hasman H, Dalsgaard A, Vieira A, Jensen LB (2008). Detection of a single isolate of CTX-M-1-producing Escherichia coli from healthy pigs in Denmark. Journal of Antimicrobial Chemotherapy 61:747-749.

Yuan L, Liu JH, Hu GZ, Pan Y-S, Liu Z-M, Mo J, Wei Y-J (2009). Molecular characterization of extended-spectrum beta-lactamase producing Escherichia coli isolates from chickens in Henan Province, China. Journal of Medical Microbiology 58:1449-1453.

Zurfluh K, Hächler H, Nüesch-Inderbinen M, Stephan R (2013). Characteristics of extended-spectrum $\beta$-lactamase- and carbapenemase-producing Enterobacteriaceae isolates from rivers and lakes in Switzerland. Applied and Environmental Microbiology 79(9):3021-3026. 